\title{
Recruitment for excellence - The German path for research university
}

Wolfram Ressel, University of Stuttgart

This manuscript is not available according to publishing restriction. Thank you for your understanding. 